ORIGINAL ARTICLE

\title{
An intensive perioperative regimen of pregabalin and celecoxib reduces pain and improves physical function scores six weeks after total hip arthroplasty: A prospective randomized controlled trial
}

\author{
Nicole ME Carmichael PhD ${ }^{1}$, Joel Katz $\mathrm{PhD}^{2}$, Hance Clarke MD PhD², Deborah Kennedy $\mathrm{PT}^{1}$, \\ Hans J Kreder MD MPH FRCSC ${ }^{3}$, Jeffrey Gollish MD FRCSC ${ }^{1}$, Colin JL McCartney MBChB FRCA FRCPC ${ }^{1}$
}

\begin{abstract}
NME Carmichael, J Katz, H Clarke, et al. An intensive perioperative regimen of pregabalin and celecoxib reduces pain and improves physical function scores six weeks after total hip arthroplasty: A prospective randomized controlled trial. Pain Res Manag 2013;18(3):127-132.
\end{abstract}

BACKGROUND: Despite the success of total hip arthroplasty (THA), some patients experience persistent pain and poor function after surgery. Predictors of poor outcomes include the presence of significant pre- and postoperative pain. Patients undergoing THA often experience severe, longstanding pain before surgery that may compromise the outcome of the procedure.

OBJECTIVES: To evaluate the effects of administering pregabalin and celecoxib for two weeks before and three weeks after THA in patients with moderate to severe pain before surgery. The aim was to determine whether patients with well-controlled pain both before surgery and in the acute postoperative period experience less pain and better physical function six weeks after THA.

METHODS: A randomized, double-blinded, placebo-controlled pilot study was conducted. Group 1 received pregabalin ( $75 \mathrm{mg}$ twice per day) and celecoxib (100 mg twice per day) for 14 days before THA and for three weeks after discharge. Group 2 received a placebo for the same duration. All patients received pregabalin and celecoxib $2 \mathrm{~h}$ before surgery and while in the hospital.

RESULTS: On the morning of surgery, patients in group 1 reported less pain at rest (mean $[ \pm \mathrm{SD}$ ] pain intensity measured on a visual analogue scale [VAS] $2.1 \pm 1.4)$ compared with group $2(3.3 \pm 1.9 ; \mathrm{P}=0.04)$. Patients in group 1 experienced less pain $3 \mathrm{~h}$ to $4 \mathrm{~h}$ postoperation $(\mathrm{P}<0.001)$. There was no difference in morphine consumption between the two groups. Six weeks after THA, movement-evoked pain was lower in group 1 (VAS 0.8 \pm 0.6 ) compared with group 2 (VAS 2.0 $\pm 1.3 ; \mathrm{P}=0.01$ ). Group 1 reported better physical function, measured using the Western Ontario and McMaster University Osteoarthritis Index questionnaire score $(\mathrm{P}=0.04)$. There was no significant difference in 6 min walk test performance between the two groups.

CONCLUSION: Intensive pain control with pregabalin and celecoxib improves pain and physical function after THA.

Key Words: Multimodal analgesia; Pre-emptive anesthesia
Un schéma posologique périopératoire intensif de prégabaline et de célécoxib réduit la douleur et améliore les indices de fonction physique six semaines après une arthroplastie totale de la hanche : un essai prospectif aléatoire et contrôlé

HISTORIQUE : Malgré le succès de l'arthroplastie totale de la hanche $(\mathrm{ATH})$, certains patients ressentent des douleurs persistantes et présentent une fonction peu satisfaisante après l'opération. Les prédicteurs d'issues médiocres incluent la présence d'une douleur préopératoire et postopératoire marquée. Les patients qui subissent une ATH souffrent souvent de douleurs importantes de longue date avant l'opération, et ces douleurs peuvent compromettre l'issue de l'intervention.

OBJECTIFS : Évaluer les effets de la prégabaline et du célécoxib administrés deux semaines avant et trois semaines après l'ATH chez des patients qui souffrent de douleur modérée à grave avant l'opération. Létude visait à déterminer si les patients dont la douleur était bien contrôlée tant avant la chirurgie que pendant la période postopératoire aiguë souffrent moins et présentent une meilleure fonction physique six semaines après l'ATH.

MÉTHODOLOGIE : Les chercheurs ont mené une étude pilote aléatoire à double insu contrôlée contre placebo. Le groupe 1 a reçu de la prégabaline (75 mg deux fois par jour) et du célécoxib (100 mg deux fois par jour) 14 jours avant l'ATH et trois semaines après le congé. Le groupe 2 a reçu un placebo pendant la même période. Tous les patients ont pris de la prégabaline et du célécoxib deux heures avant l'opération et pendant l'hospitalisation. RÉSULTATS : Le matin de l'opération, les patients du groupe 1 ont déclaré moins de douleur au repos (intensité de la douleur moyenne [ $\pm E ́ T]$ mesurée sur une échelle visuelle analogique [ÉVA] $2,1 \pm 1,4)$ que le groupe 2 $(3,3 \pm 1,9 ; \mathrm{P}=0,04)$. Les patients du groupe 1 ressentaient moins de douleur de trois à quatre heures après l'opération $(\mathrm{P}<0,001)$. Il n'y avait pas de différence de consommation de morphine entre les deux groupes. Six semaines après l'ATH, la douleur évoquée par le mouvement était plus faible dans le groupe 1 (ÉVA $0,8 \pm 0,6$ ) que dans le groupe 2 (ÉVA 2,0 0 1,3; $\mathrm{P}=0,01$ ). Le groupe 1 a déclaré une meilleure fonction physique, mesurée selon le score du questionnaire sur l'indice d'arthrose $(\mathrm{P}=0,04)$ des universités Western Ontario et McMaster. Il n'y avait pas de différence significative entre les deux groupes dans l'épreuve à l'effort de 6 minutes.

CONCLUSION : Le contrôle intensif de la douleur à l'aide de prégabaline et de célécoxib atténue la douleur et améliore la fonction physique après une $\mathrm{ATH}$.

pain and improve physical function following many different surgical procedures (4-12). Previous studies have investigated the effects of administering these medications $1 \mathrm{~h}$ to $2 \mathrm{~h}$ before surgery and from one to up to 14 days after surgery (6-12). To date, no study has investigated the effects of administering a multimodal regimen well before and continuing for at least three weeks after THA.

The presence of pre-existing chronic pain may amplify central sensitization that can occur during and after the surgical incision (13). Therefore, for patients with moderate to severe pain before surgery, it alone or in conjunction with celecoxib, have been shown to reduce

${ }^{1}$ Department of Anesthesia, Sunnybrook Holland Orthopedic and Arthritic Centre; ${ }^{2}$ University of Toronto, Toronto General Hospital;

${ }^{3}$ Sunnybrook Health Sciences Centre, Toronto, Ontario

Correspondence: Dr Colin JL McCartney, Department of Anesthesia, Sunnybrook Holland Orthopedic and Arthritic Centre, 40 Wellesley Street,

Toronto, Ontario M5S 1A8. Telephone 416-967-8500, fax 416-967-8593, e-mail colin.mccartney@utoronto.ca 
may be necessary to administer a multimodal regimen well before surgery in addition to during the intra- and postoperative period when nociceptive inputs are maximal. The present study evaluated the effects of controlling pain using pregabalin and celecoxib for two weeks before and during hospitalization and for three weeks after THA on pain and physical function six weeks postoperatively. This regimen was compared with the standard perioperative pain protocol used at our institution, which consisted of administering pregabalin and celecoxib $2 \mathrm{~h}$ before surgery and for the duration of the hospital stay.

\section{METHODS}

The present study was approved by the Research Ethics Board and Health Canada (NCT00581685). Written informed consent was obtained from each patient. Patients were eligible for inclusion if they were between 18 and 80 years of age and scheduled for THA, with pain intensity scores (measured on a visual analogue scale [VAS]) $\geq 4$. Exclusion criteria included: patients taking $>30 \mathrm{mg}$ morphine/day; rheumatoid arthritis; psychiatric disorders; impaired renal function; body mass index $>40 \mathrm{~kg} / \mathrm{m}^{2}$; and patients unable to use patient-controlled analgesia (PCA).

\section{Randomization and study medication}

A computer-generated randomization schedule was used to assign patients to one of two treatment groups. The schedule was created by the hospital investigational pharmacy, which was otherwise not involved in the clinical care of patients or in the conduct of the present trial. Pregabalin, celecoxib and placebo medications were prepared and dispensed by the Sunnybrook Health Sciences Centre Investigational Pharmacy (Toronto, Ontario) to maintain doubleblinded conditions. The capsules were packaged into vials that were labelled 'pregabalin or placebo' and 'celecoxib or placebo'.

\section{Treatment protocol}

All patients scheduled to undergo THA between March 2008 and March 2009 were screened for recruitment into the present study. Patients were recruited at their preoperative assessment visit at least two weeks before surgery and were randomly assigned to one of two treatment groups. Group 1 (G1) received pregabalin (75 mg twice per day) and celecoxib (100 mg twice per day) for two weeks before surgery and for three weeks from the day of discharge. Group 2 (G2) received identical placebo tablets for the same period of time. Before beginning the study medication, patients in both groups were instructed to discontinue taking other pain medication. One week after starting the study medication, patients with inadequate pain relief (VAS pain score $>4$ ) were instructed to increase their dose of medication labelled 'pregabalin or placebo' to $75 \mathrm{mg}$ three times per day. If patients in either group experienced intolerable side effects from the higher dose of 'pregabalin or placebo' they were instructed to return to the previous lower dosage. All patients were permitted to use acetaminophen/codeine (Tylenol I, II or III, Johnson and Johnson, USA) or acetaminophen/oxycodone (Percocet, Endo Pharmaceuticals, USA) before surgery if the study medication did not provide them with adequate pain relief. Patients were also provided with diaries with instructions to report daily their pain at rest and with movement $(0=$ no pain, $10=$ worst most imaginable pain $)$, sleep quality, side effects, use of additional pain medication (dose and number of tablets) and compliance with the study medication.

All patients received the same opioid and nonopioid medications during the pre-, intra- and postoperative periods. Two hours before surgery, all patients received $400 \mathrm{mg}$ celecoxib, $150 \mathrm{mg}$ pregabalin and $1 \mathrm{~g}$ of acetaminophen. Patients were sedated with midazolam and received a spinal anesthetic for surgery. Postoperatively, all patients received pregabalin (75 $\mathrm{mg}$ twice per day), celecoxib (200 mg twice per day) and acetaminophen ( 1 g every $6 \mathrm{~h}$ ) for five days. PCA morphine was available for the first $48 \mathrm{~h}$ after surgery and was set to deliver morphine at $1 \mathrm{mg}$ per demand with a 5 min lockout. Patients were instructed to maintain a VAS pain score of between 2 and 4 . If the VAS pain score was $\geq 4$ at rest on two consecutive occasions, the dose of PCA morphine was increased to $1.5 \mathrm{mg}$ per demand. Following discontinuation of the PCA pump, patients received oxycodone $(5 \mathrm{mg}$ every $2 \mathrm{~h}$ pro re nata) on request for pain relief. Sedation, nausea, pruritus, dizziness and vomiting was assessed immediately after surgery and twice daily until discharge. On the day of discharge, patients were instructed to resume taking their preoperative study medication for three weeks and were prescribed acetaminophen/oxycodone (5 mg/375 mg) as needed for breakthrough pain. Patients were discharged home with diaries identical to those provided before surgery.

\section{Assessment of pain, physical function and psychosocial factors}

Pain scores at rest and movement were assessed using a VAS $(0=$ no pain; 10 = worst pain). Patients were administered three questionnaires to evaluate self-reported measures of pain, physical function and psychosocial outcomes: the Western Ontario and McMaster University Osteoarthritis Index (WOMAC) (14), the Pain Disability Index (15) and the Hospital Anxiety and Depression Scale (16). These outcomes were evaluated at baseline, one week after patients started their study medication, on the morning before surgery and at weekly intervals from one to six weeks after THA (Figure 1). Physical function was also evaluated using the 6 min walk test. The test was conducted by instructing patients to walk at a comfortable pace for 6 min around a $47 \mathrm{~m}$ track. Patients completed the 6 min walk test at baseline, on the day of surgery and six weeks postoperatively.

\section{Sample size estimation}

A total of 80 patients were expected to be enrolled in the study. This sample size estimation was based on findings from a previous study that identified a mean $( \pm$ SD) difference of $36 \pm 50 \mathrm{~m}$ walked in 6 min to produce a meaningful impact on patients six weeks after surgery (17). Based on this value, the study determined that a total of 64 patients ( 32 per group) would be required for $80 \%$ power and a risk of type I error at $5 \%$. This sample size estimate was also used for the present study and, based on a $20 \%$ attrition rate (ie, drop outs, patient withdrawal) for the primary six-week end point, it was determined that approximately 80 patients should be recruited.

\section{Statistical analysis}

Data analyses were performed by an independent blinded statistician. The primary outcome of the present study was physical function assessed by 6 min walk test at six weeks following surgery. Secondary outcome measures included pain, physical function and psychosocial factors. Demographic and clinical variables were compared using ANOVA and Fisher's exact test. The proportion of patients who reported adverse effects and required additional pain medication were compared between the two groups using Fisher's exact test. Primary and secondary outcomes were analyzed using a two-way repeated-measures ANOVA. The analysis was performed on an intention-to-treat basis. A planned analysis comparing treatment groups six weeks postoperatively was performed for all outcomes; $\mathrm{P}<0.05$ was considered to be statistically significant.

\section{RESULTS}

\section{Recruitment and retention of patients}

Forty-seven patients were enrolled in the study, with 23 patients assigned to G1 (pregabalin/celecoxib) and 24 patients assigned to G2 (placebo/placebo).

A total of 31 patients completed the study until the six-week postoperative time point ( $n=15$ in G1 and $n=16$ in G2) (Figure 2). In G1, eight patients did not complete the study. Of these, four withdrew before beginning the study medication, two did not complete the study due to intolerable adverse effects (dizziness) from the study medication, one patient died three weeks post-THA due to a surgical complication and one patient was withdrawn due to a protocol violation (patient had an American Society of Anesthesiologists physical status classification of IV). In G2, eight patients did not complete the study. Of these, six patients withdrew before beginning the study medication, one patient withdrew due to inadequate pain control and one patient was removed who requested a general anesthetic instead of a spinal anesthetic on the day of surgery. 


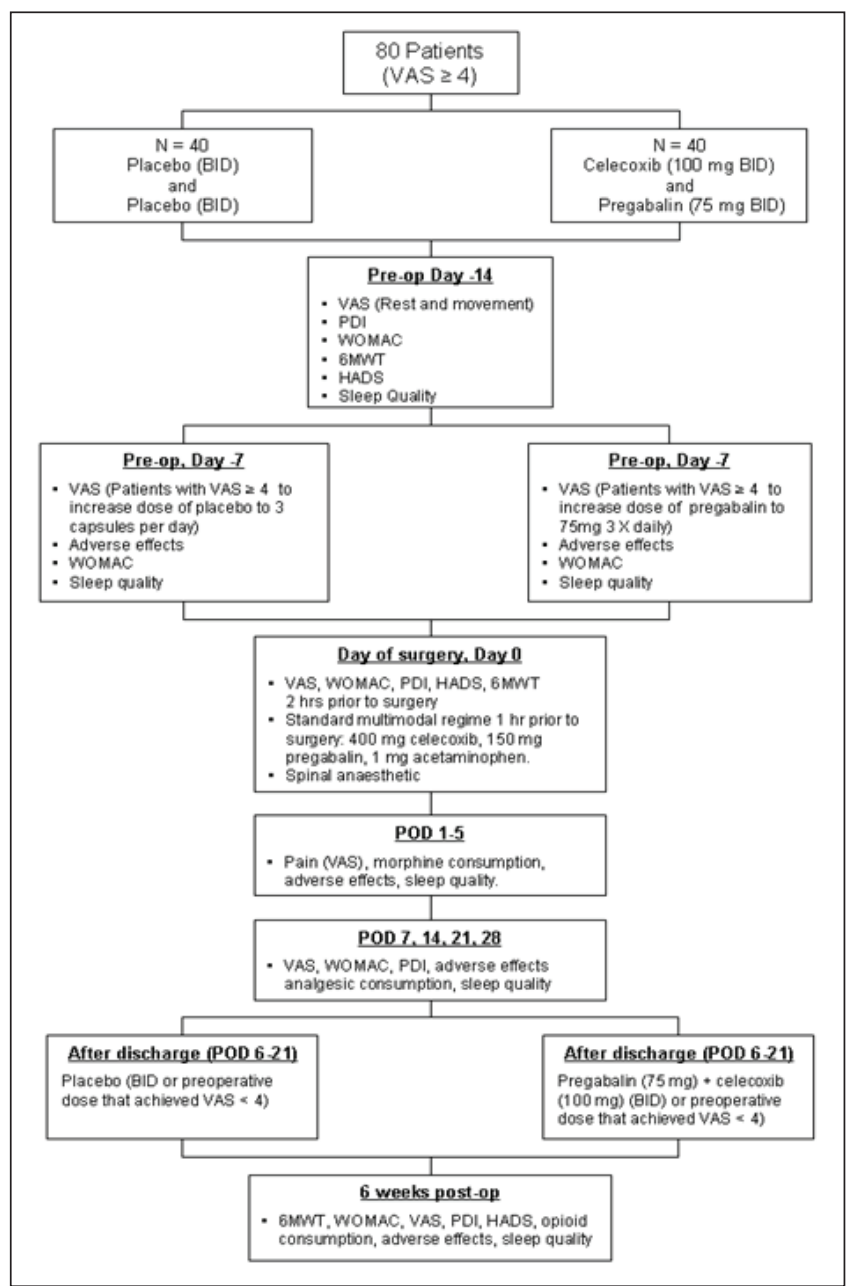

Figure 1) Flow chart showing timing of events and study design. 6MWT $6 \mathrm{~min}$ walk test; BID Twice per day; HADS Hospital Anxiety and Depression Scale; PDI Pain Disability Index; POD Postoperative day; Post-op Postoperative; Pre-op Preoperative; VAS Visual analogue scale; WOMAC Western Ontario and McMaster University Osteoarthritis Index

The proposed sample size of 80 patients could not be recruited because the study was put on hold by the Research Ethics Board for approximately six months following the death of a study participant. During this time, an investigation was conducted and concluded that the death was not related to the study. An interim analysis of the primary outcome measure (6 min walk test) based on the 31 patients who had completed the study was performed by a statistician who was blinded to the treatment allocation. The analysis indicated that an additional 200 patients ( 100 patients per group) would be needed to detect a significant difference in the $6 \mathrm{~min}$ walk test between the two groups. Due to insufficient funds to recruit 200 additional patients, the study was not restarted; rather, the data from the 31 patients who had completed the study were analyzed.

\section{Demographic and clinical variables}

Table 1 summarizes the demographic and clinical variables describing the sample of 31 patients who completed the study. The groups were comparable with respect to age, sex, body mass index, American Society of Anesthesiologists physical status classifications and duration of surgery. The duration of pre-existing pain was not an inclusion criterion, although all patients experienced severe to moderate pain for at least six months before surgery.

\section{Intervention}

Efficacy and side effect profile: Within the first $24 \mathrm{~h}$ of starting the study medication, the proportion of patients experiencing adverse effects was significantly greater in G1 (12/18) compared with G2

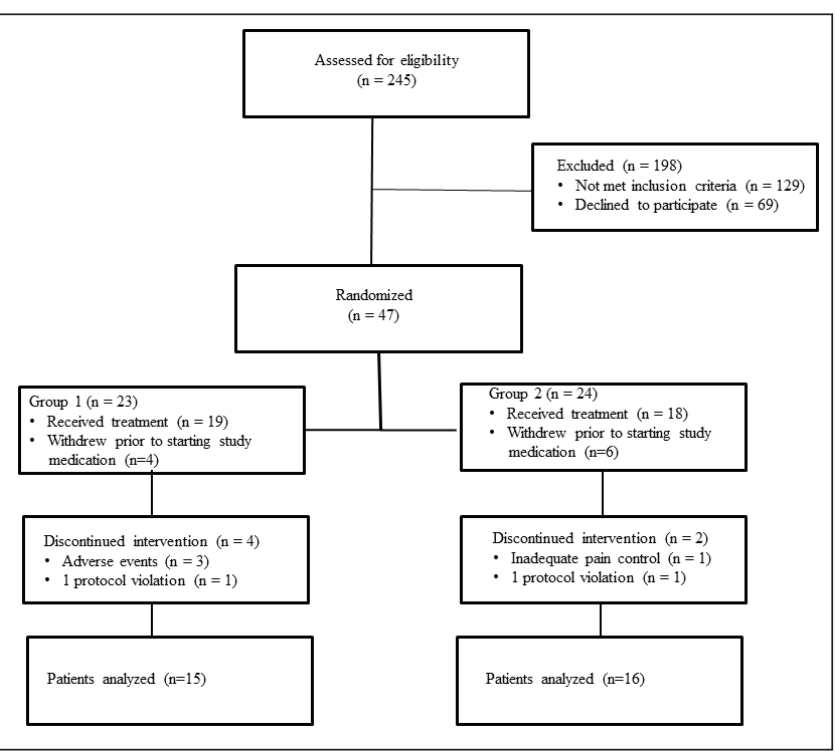

Figure 2) Number of patients randomized, withdrawn and completing the study until the six-week postoperative time point

\section{TABLE 1}

Demographic and clinical variables

\begin{tabular}{lcc}
\hline Variable & G1 $(\mathbf{n}=\mathbf{1 8})$ & $\mathbf{G 2}(\mathbf{n}=\mathbf{1 8})$ \\
\hline Age, years & $59.1 \pm 10.1$ & $61.3 \pm 15.1$ \\
Sex, male/female, $\mathrm{n} / \mathrm{n}$ & $10 / 8$ & $12 / 6$ \\
Body mass index, $\mathrm{kg} / \mathrm{m}^{2}$ & $28.5 \pm 5.1$ & $29.8 \pm 4.8$ \\
ASA classification, $\mathrm{n}$ & & \\
I & 5 & 6 \\
II & 11 & 10 \\
III & 3 & 2 \\
Duration of surgery, min & $148.8 \pm 32.8$ & $147.4 \pm 27$ \\
\hline
\end{tabular}

Data presented as mean \pm SD unless otherwise indicated. Data from patients who were randomized but withdrew before starting study medication or were removed due to protocol violation (Group 1 [G1] [pregabalin/celecoxib], $n=5$; Group 2 [G2] [placebo/placebo], $n=6$ ) were not included. No significant differences were observed between groups. ASA American Society of Anesthesiologists

\section{TABLE 2}

Side effect profile and additional analgesic requirements before surgery

\begin{tabular}{|c|c|c|}
\hline Pain medication & G1 (n=16) & $\mathrm{G} 2(n=18)$ \\
\hline $\begin{array}{l}\text { Patients requiring additional pain medication } \\
\text { before surgery }\end{array}$ & 4 & 6 \\
\hline $\begin{array}{l}\text { Number of patients who increased dose of study } \\
\text { medication to three times per day }\end{array}$ & 10 & 16 \\
\hline Side effects & G1 $(n=18)$ & G2 $(n=18)$ \\
\hline Sedation & 4 & 2 \\
\hline Dizziness & 7 & 2 \\
\hline Blurred vision & 1 & 0 \\
\hline
\end{tabular}

Data presented as n. Two patients in group 1 (G1) (pregabalin/celecoxib) experienced severe dizziness and withdrew from the study. No significant differences were observed between groups. G2 Group 2 (placebo/placebo)

(4/18) $(\mathrm{P}<0.05)$ (Table 2). Two patients in G1 experienced intolerable side effects (dizziness) and withdrew from the study. One week after beginning the study (preoperative day 7), 63\% (10/16) of patients in G1 and $89 \%(16 / 18)$ of patients in G2 continued to experience inadequate pain relief (VAS $>4$ ) and increased the dose of pregabalin/placebo to $75 \mathrm{mg}$ three times daily. In G1, seven patients reported less pain within two days of increasing their dose of pregabalin and three patients failed to achieve any additional pain relief. No 
TABLE 3

Pain, physical function and psychosocial factors

\begin{tabular}{|c|c|c|c|c|c|c|}
\hline & \multirow[b]{2}{*}{ Group } & \multicolumn{2}{|c|}{ Pain intensity } & \multirow[b]{2}{*}{ WOMAC } & \multirow[b]{2}{*}{ Pain Disability Index } & \multirow[b]{2}{*}{ HADS } \\
\hline & & VAS-R & VAS-M & & & \\
\hline \multirow[t]{2}{*}{ Baseline } & 1 & $4.0 \pm 2.3$ & $7.3 \pm 1.6$ & $47.3 \pm 16.3$ & $32.2 \pm 18.0$ & $10.6 \pm 6.5$ \\
\hline & 2 & $3.6 \pm 2.1$ & $7.6 \pm 1.4$ & $51.7 \pm 11.6$ & $33.1 \pm 16.1$ & $8.3 \pm 4.3$ \\
\hline Preoperative day 7 & 2 & $2.7 \pm 2.3$ & $6.6 \pm 1.7$ & $49.8 \pm 13.4$ & NC & NC \\
\hline \multirow[t]{2}{*}{ Day of surgery } & 1 & $2.1 \pm 1.4^{*}$ & $4.8 \pm 2.4$ & $43.3 \pm 16.9$ & $27.3 \pm 21.8$ & $8.7 \pm 6.6$ \\
\hline & 2 & $3.3 \pm 1.9^{*}$ & $5.8 \pm 1.9$ & $50.5 \pm 11.9$ & $34.2 \pm 11.9$ & $4.3 \pm 3.8$ \\
\hline Postoperative day 7 & 2 & $2.9 \pm 2.1$ & $4.8 \pm 1.9$ & $35.7 \pm 12.5$ & $39.8 \pm 12.6$ & NC \\
\hline \multirow[t]{2}{*}{ Postoperative day 14} & 1 & $0.4 \pm 0.7$ & $1.6 \pm 1.3$ & $19.2 \pm 5.4$ & $33.4 \pm 14.0$ & NC \\
\hline & 2 & $1.7 \pm 2.6$ & $3.7 \pm 2.8$ & $30.9 \pm 11.8$ & $37.3 \pm 12.6$ & NC \\
\hline \multirow[t]{2}{*}{ Postoperative day 21} & 1 & $0.4 \pm 0.5$ & $1.1 \pm 1.0$ & $17.1 \pm 8.4$ & $32.1 \pm 19.1$ & NC \\
\hline & 2 & $0.3 \pm 0.5$ & $2.5 \pm 0.8$ & $27.6 \pm 11.5$ & $32.3 \pm 16.7$ & NC \\
\hline Postoperative day 28 & 1 & $0.0 \pm 0.2$ & $0.7 \pm 0.9$ & $16.9 \pm 7.0$ & $29.2 \pm 17.0$ & NC \\
\hline
\end{tabular}

Data presented as mean $\pm S D$. *Indicates time points that are significantly different between Group 1 (G1) (pregabalin/celecoxib) versus Group 2 (G2) (placebo/ placebo). Day of surgery refers to the day of surgery in the morning before total hip arthroplasty. HADS Hospital Anxiety and Depression Scale; NC Not completed; VAS-M Visual analogue scale score with movement; VAS-R Visual analogue scale score at rest; WOMAC Western Ontario and McMaster University Osteoarthritis Index

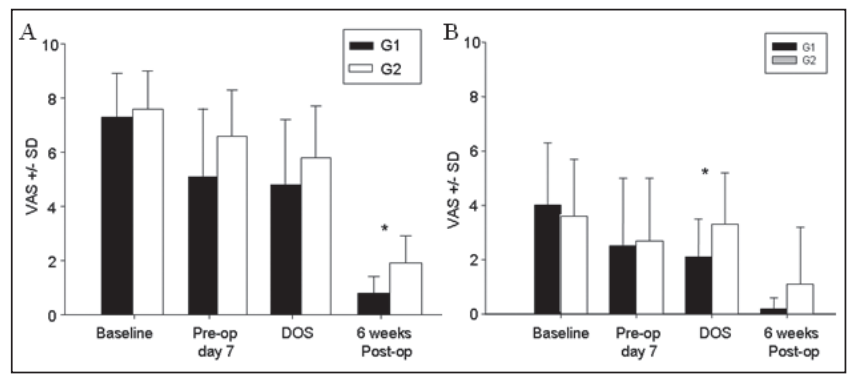

Figure 3) A Movement-evoked pain at baseline, one week after starting study medication (preoperative day 7), on the day of surgery (DOS) and at six weeks after total hip arthroplasty. Data presented as mean \pm SD score on a visual analogue scale (VAS). Patients who received pregabalin and celecoxib for two weeks before and three weeks after surgery (Group 1 [G1]) had significantly less pain with movement six weeks postoperatively compared with patients who recieved placebo (Group $2[\mathrm{G} 2])(* P<0.05)$. B Resting pain at baseline, one week after starting study medication (preoperative day 7), on the DOS and six weeks after total hip arthroplasty. Patients who received pregabalin and celecoxib for two weeks before surgery had significantly less pain at rest on the DOS $(* \mathrm{P}<0.05)$. Pre-op Preoperative; Post-op Postoperative

adverse effects were reported. The number of patients using additional pain medication (acetaminophen/codeine or acetaminophen/ oxycodone) was similar in both groups (Table 2).

The number of patients who experienced an adverse event within the first $48 \mathrm{~h}$ after surgery was significantly different between the two groups $(\mathrm{P}<0.05)$. Forty-four per cent $(7 / 16)$ of patients in the placebo group reported at least one adverse event (sedation $n=4$, dizziness $\mathrm{n}=6$, nausea $\mathrm{n}=5$, vomiting $\mathrm{n}=2$ ). Patients who received active treatment before surgery did not report any adverse effects while in hospital.

Pain, physical function and psychosocial outcomes: There were no significant differences in pain and physical function scores between the two groups at baseline or one week after starting the study medication (Table 3). On the morning of surgery, patients in G1 reported less pain at rest (VAS $=2.1 \pm 1.4 ; n=16)$ compared with G2 $(\mathrm{VAS}=3.3 \pm 1.9 ; \mathrm{n}=16)(\mathrm{P}=0.04)$. Postoperatively, pain scores were lower in $\mathrm{G} 1$ at $3 \mathrm{~h}(1.0 \pm 1.1)$ and $4 \mathrm{~h}(1.3 \pm 1.0)$ compared with patients in $\mathrm{G} 2(3 \mathrm{~h}=2.9 \pm 1.8 ; 4 \mathrm{~h}=4.3 \pm 2.3 ; \mathrm{P}=0.001)$. On postoperative days 1 through 5 , there was no difference in pain scores between the two groups. There was also no difference in cumulative morphine consumption at $48 \mathrm{~h}$ (G1 144.2 $\pm 85 \mathrm{mg} ; \mathrm{G} 2177.6 \pm 101 \mathrm{mg} ; \mathrm{P}=0.32$ ). Six weeks after surgery, patients in $\mathrm{G} 1$ reported less pain with movement $(\mathrm{P}=0.01$; $\mathrm{n}=15$ ) (Figures $3 \mathrm{~A}$ and $3 \mathrm{~B}$ ) and better physical function measured using WOMAC scores $(\mathrm{P}=0.02 ; n=15)$ compared with $\mathrm{G} 2(\mathrm{n}=16)$ (Figure 4$)$. There was no difference in 6 min walk test performance between the two groups at baseline (G1 369.3 $\pm 143.2 \mathrm{~m}$; G2 378.5 $\pm 133.6 \mathrm{~m}$ ), the day of surgery $(\mathrm{G} 1,370.6 \pm 146.7 \mathrm{~m} ; \mathrm{G} 2,375.5 \pm 138.1 \mathrm{~m})$ or six weeks after THA (G1, 397 $\pm 76.6 \mathrm{~m} ; \mathrm{G} 2,397.5 \pm 83.9 \mathrm{~m} ; \mathrm{P}>0.05)$.

\section{DISCUSSION}

The results of the present study demonstrate that administering pregabalin and celecoxib for two weeks before surgery and for three weeks postoperatively in the context of an in-hospital multimodal regimen with spinal anesthesia improves pain six weeks after THA. Although we did not find a significant difference in the 6 min walk test, patients who received pregabalin and celecoxib had significantly better physical function scores on the WOMAC six weeks postoperatively. Because we were unable to complete the study with the proposed sample size, it is likely that our study was underpowered to detect a difference in 6 min walk test performance.

We designed our study with the intent to reduce the degree of central sensitization caused by pre-existing pain before surgery in addition to that evoked by the surgical trauma and inflammatory response that can ensue for weeks after. We chose a two-week preoperative regimen to allow sufficient time for patients to experience pain reduction and to titrate their dose. However, it may be necessary to use higher doses of pregabalin than those used in the present study because some patients in the pregabalin and celecoxib group $(n=3)$ still experienced moderate pain after increasing their dose of pregabalin. Previous studies have shown that the minimum effective dose of pregabalin is between $225 \mathrm{mg} /$ day and $300 \mathrm{mg}$ /day $(1,2)$. This dose is associated with a significantly higher incidence of dizziness and sedation. Although a much lower dose of pregabalin was used in the present study, two patients in the active treatment group withdrew within $24 \mathrm{~h}$ of starting their study medication due to severe dizziness. This represents a limitation to the use of pregabalin, even at the lower doses used in the present study. 
It is unclear from our findings whether a three-week postoperative regimen is the optimal duration required to improve pain and functional outcomes in the long term. A recent study found that administering pregabalin for only 14 days after knee arthroplasty improved function at 30 days postoperatively in addition to reducing neuropathic pain at three and six months after surgery (4). Additional studies are needed to delineate the optimal duration and dose of pregabalin.

Because our protocol was designed to evaluate the effects of pregabalin and celecoxib across the perioperative period, we were unable to infer from our results whether both the pre- and postoperative interventions are equally responsible, or necessary, for the improvements we observed. It is also unclear whether both drugs were responsible for the effects we observed since we did not evaluate the efficacy of either drug alone. This is a limitation of the study that could be improved by evaluating the effects of altering the timing and combination of the regimen.

Other studies have questioned the benefit of preoperative administration of pregabalin for acute postoperative pain (18-21). However, we found significant differences in pain scores at $3 \mathrm{~h}$ and $4 \mathrm{~h}$ after surgery, suggesting that the two-week preoperative intervention with pregabalin and celecoxib reduced early acute postoperative pain.

Previous studies have shown that for patients to experience 'much improvement', they need at least a 70\% reduction in pain, and a pain reduction of $30 \%$ is perceived as minimal (22-25). In the present study, patients in G1 reported a $71 \%$ reduction in VAS scores on the day of surgery compared with $56 \%$ in G2. Similar reductions were also found six weeks postoperatively. Therefore, the reduction in pain scores may be both statistically and clinically significant.

A minimum change in WOMAC scores of $36 \%$ to $40 \%$ is required to be clinically meaningful in patients with arthritis $(26,27)$. In the present study, patients in $\mathrm{G} 1$ had a $70 \%$ reduction and $\mathrm{G} 2 \mathrm{had}$ a $53 \%$ reduction in cumulative WOMAC scores from baseline to six weeks postoperatively. This represents an overall difference of $17 \%$ between the two groups. Therefore, the difference in WOMAC scores between the two groups was statistically significant but unlikely to be clinically meaningful. It must be noted, however, that it may be misleading in this context to put too much emphasis on interpreting this between-group difference in score as the measure of clinical significance. Because $\mathrm{G} 2$ received pregabalin and celecoxib while in hospital, it does not represent a true placebo control condition, which would have received the placebo throughout the three phases of the study. Presumably, a true placebo control group would have demonstrated a smaller reduction in cumulative WOMAC scores than the $53 \%$ observed in G2, thereby leading to a larger difference in score than the $17 \%$ we observed between G1 and G2. Whether this difference would approach the 36\% to $40 \%$ required for clinical significance could not be determined by the present study.

Patients receiving pregabalin and celecoxib did not report any dizziness or sedation after surgery, whereas $44 \%$ of patients in the control group experienced at least one adverse effect that may have been opioid-related or due to starting pregabalin before surgery. It is possible that patients in the active treatment group experienced less pregabalin-related side effects in the postoperative period due to commencing and titrating pregabalin two weeks before surgery. Initiating pregabalin treatment several days before surgery (as in G1) may be a useful strategy in the orthopedic population that requires early rehabilitation.

In conclusion, an intensive pain control regimen with pregabalin and celecoxib before and after total hip replacement surgery may improve pain and physical function. Replication with a larger sample size is recommended to confirm the benefits of this intervention.

FUNDING: Physician Services Incorporated. Joel Katz is supported by a CIHR Canada Research Chair in Health Psychology.

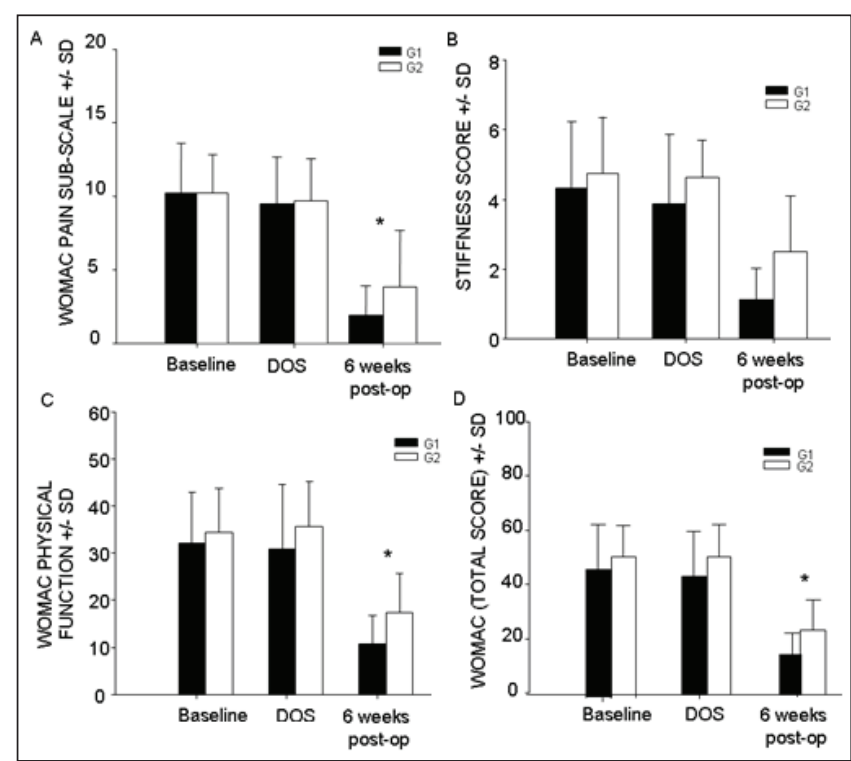

Figure 4) Western Ontario and McMaster University Osteoarthritis Index (WOMAC) scores at baseline, on the day of surgery (DOS) and six weeks after total hip arthroplasty. Data presented as mean \pm SD. Six weeks postoperatively (post-op), patients who received pregabalin and celecoxib (Group 1 [G1]) for two weeks before and three weeks after surgery scored significantly better on the pain (A), stiffness (B) and physical function (C) subsections of the WOMAC compared with patients who received placebo (Group 2 [G2]) for the same length of time. Cumulative WOMAC scores were also significantly improved six weeks postoperatively in patients who received active treatment compared with the placebo group (D). A lower WOMAC score indicates less pain and stiffness and better physical function. $* P<0.05$.

\section{REFERENCES}

1. Goldstein DH, Ellis J, Brown R, et al. Recommendations for improved acute pain services: Canadian collaborative acute pain initiative. Pain Res Manag 2004;9:123-30.

2. Kalkman CJ, Visser K, Moen J, Bonsel GJ, Grobbee DE, Moons KG. Preoperative prediction of severe postoperative pain. Pain 2003;105:415-23.

3. Katz J, Seltzer Z. Transition from acute to chronic postsurgical pain: Risk factors and protective factors. Expert Rev Neurother 2009;9:723-44.

4. Buvanendran A, Kroin JS, Della Valle CJ, Kari M, Moric M, Tuman KJ. Perioperative oral pregabalin reduces chronic pain after total knee arthroplasty: A prospective, randomized, controlled trial. Pain Med 2010;110:199-207.

5. Katz J, Clarke H, Seltzer Z. Review article: Preventive analgesia: Quo vadimus? Anesth Analg 2011;113:1242-53.

6. Clarke C, Pereira S, Kennedy D, et al. Gabapentin decreases morphine consumption and improves functional recovery following total knee arthroplasty. Pain Res Manag 2009;14:217-22.

7. Fassoulaki A, Stamatakis E, Petropoulos G, Siafaka I, Hassiakos D, Sarantopoulos C. Gabapentin attenuates late but not acute pain after abdominal hysterectomy. Eur J Anaesthesiol 2006;23:136-41.

8. Gilron I, Orr E, Tu D, O’Neill JP, Zamora JE, Bell AC.

A placebo controlled randomized clinical trial of perioperative administration of gabapentin, rofecoxib and their combination for spontaneous and movement-evoked pain after abdominal hysterectomy. Pain 2005;113:191-200.

9. Menigaux C, Adam F, Guignard B, et al. Preoperative gabapentin decreases anxiety and improves early functional recovery from knee surgery. Anes Analg 2005;100:1294-9.

10. Pandey CK, Priye S, Singh S, Singh U, Singh RB, Singh PK. Preemptive use of gabapentin significantly decreases postoperative pain and rescue analgesic requirements in laparoscopic cholecystectomy. Can J Anaesthes 2004;51:358-63.

11. Post Z, Restrepo C, Kahl LK, van de Leur T, Purtill JJ, Hozack WJ. Prospective evaluation of 2 different pain management protocols for total hip arthroplasty. J Arthroplasty 2010;25:410-5. 
12. Parsa AA, Sprouse-Blum AS, Jackowe DJ, Lee M, Oyama J, Parsa FD. Combined preoperative use of celecoxib and gabapentin in the management of postoperative pain. Aesthetic Plast Surg 2009;33:98-103.

13. Katz J, Seltzer Z. Transition from acute to chronic postsurgical pain: Risk factors and protective factors. Expert Rev Neurother 2009;9:723-44.

14. Kennedy DM, Stratford PW, WesselJ, Gollish JD, Penney D. Assessing stability and change of four performance measures: A longitudinal study evaluating outcome following total hip and knee arthroplasty. BMC Musculoskel Disord 2005;6:1-12.

15. Tait RC, Pollard CA, Margolis RB, Duckro PN, Krause SJ The pain disability index: Psychometric and validity data. Arch Phys Med Rehabil 1987;68:438-41.

16. Bjelland I, Dahl AA, Haug TT, Neckelmann D. The validity of the Hospital Anxiety and Depression Scale. An updated literature review. J Psychosom Res 2002;52:69-77.

17. Carli F, Mayo N, Klubien K, Schricker T, Trudel J, Belliveau P. Epidural analgesia enhances functional exercise capacity and health-related quality of life after colonic surgery: Results of a randomized trial. Anesthesiology 2002;97:540-9.

18. Paech MJ, Goy R, Chua S, Scott K, Christmas T, Doherty DA. A randomized, placebo-controlled trial of preoperative oral pregabalin for postoperative pain after minor gynecological surgery. Anesth Analg 2007;105:1449-53.

19. White PF, Tufanogullari B, Taylor J, Klein K. The effect of pregabalin on anxiety and sedation levels: A dose-ranging study. Anesth Analg 2009;108:1140-5.
20. Chang SH, Lee HW, Kim HK, Kim SH, Kim DK. An evaluation of perioperative pregabalin for prevention and attenuation of postoperative shoulder pain after laparoscopic cholecystectomy. Anesth Analg 2009;109:1284-6.

21. Jokela R, Ahonen J, Tallgren M, Haanpa“a” M, Korttila K. Premedication with pregabalin 75 or $150 \mathrm{mg}$ with ibuprofen to control pain after day-case gynaecological laparoscopic surgery. Br J Anaesth 2008;100:834-40.

22. Farrar JT, Young JP Jr, LaMoreaux L, Werth JL, Poole RM. Clinical importance of changes in chronic pain intensity measured on an 11-point numerical pain rating scale. Pain 2001;94:149-58.

23. Forouzanfar T, Weber WE, Kemler M, van Kleef M. What is a meaningful pain reduction in patients with complex regional pain syndrome type 1 . Clin J Pain 2003;19:281-5.

24. Kelly DJ, Ahmad M, Brull SJ. Preemptive analgesia II: Recent advances and current trends. Can J Anaesth 2001;48:1091-101.

25. Sloman R, Wruble AW, Rosen G, Rom M. Determination of clinically meaningful levels of pain reduction in patients experiencing acute postoperative pain. Pain Manag Nurs 2006;7:153-8.

26. Goldsmith CH, Boers M, Bombardier C, Tugwell P. Criteria for clinically important changes in outcomes: Development, scoring and evaluation of rheumatoid arthritis patient and trial profiles. OMERACT Committee. J Rheumatol 1993;20:561-5.

27. Pua YH, Cowan SM, Wrigley TV, Bennell KL. Discriminant validity of the Western Ontario and McMaster Universities Osteoarthritis Index physical functioning subscale in community samples with hip osteoarthritis. Arch Phys Med Rehabil 2009;90:1772-7. 


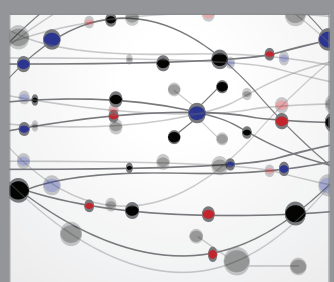

The Scientific World Journal
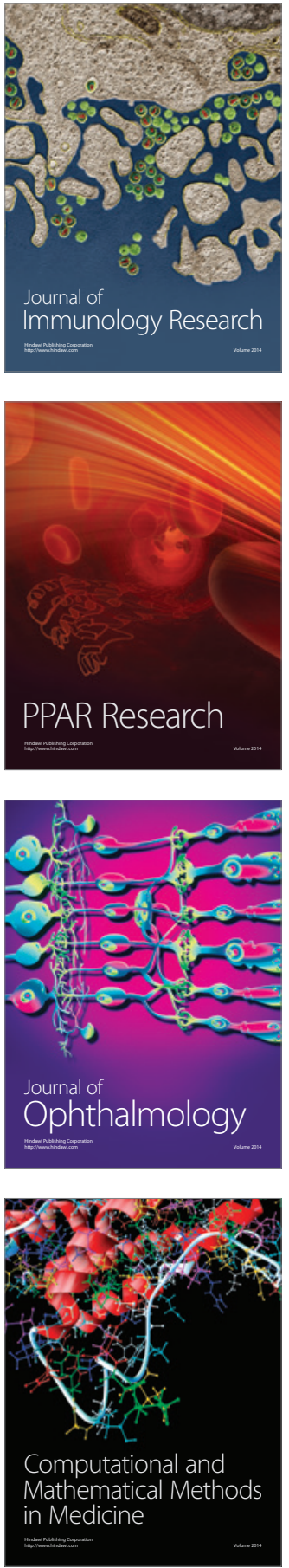

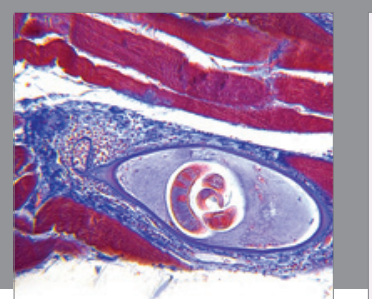

Gastroenterology Research and Practice

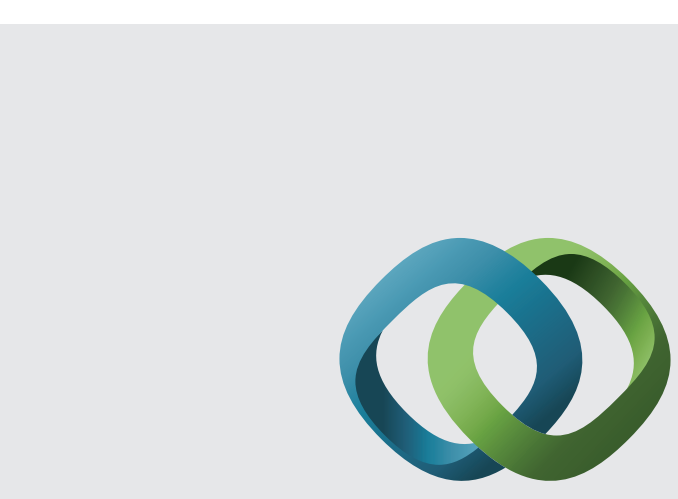

\section{Hindawi}

Submit your manuscripts at

http://www.hindawi.com
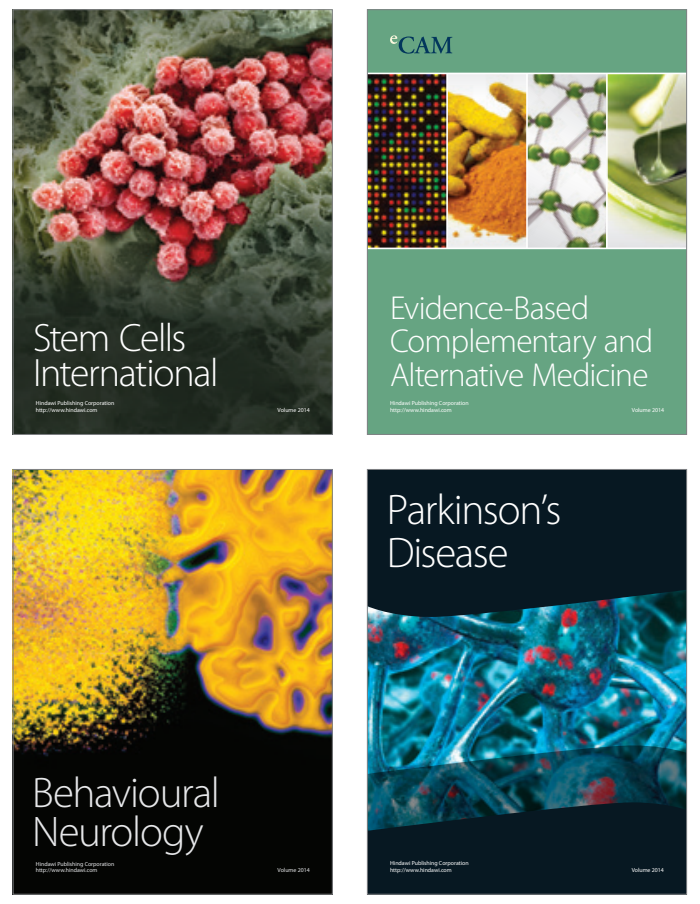
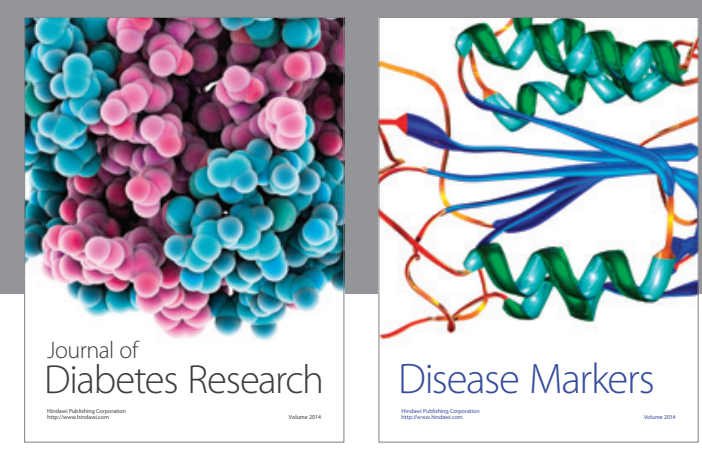

Disease Markers
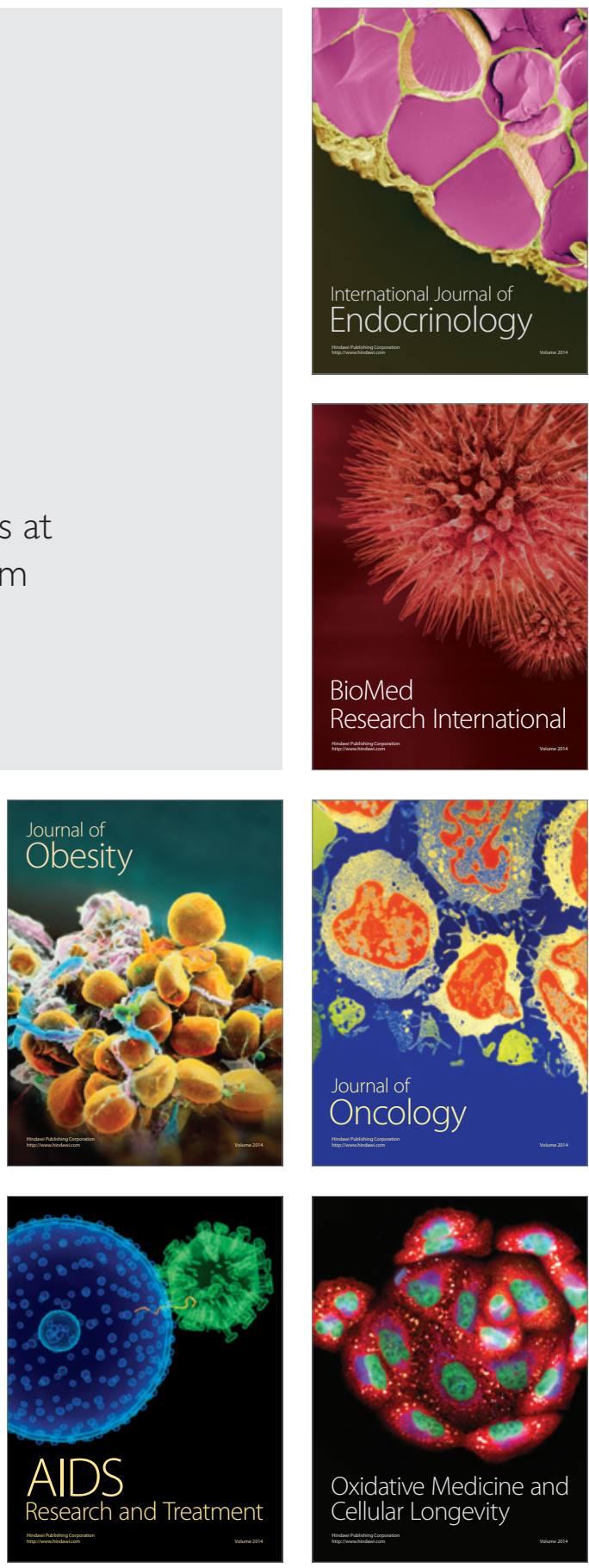